\title{
EXPERIENCIANDO O DESIGN MULTISSENSORIAL DOS PERFUMES: UM ESTUDO DO IMPACTO SENSORIAL NA INTENÇÃO DE COMPRA
}

Juliana Hollerbach de Aguilar, Msc.

Escola de Design/UEMG

hollerju@gmail.com

Cid Gonçalves Filho, Dr.

Universidade FUMEC

cid@fumec.br

Resumo: Partindo do princípio que a sinergia sensorial pode dobrar o efeito da comunicação entre o produto e o consumidor apontada pelos estudos de consumo de experiência, este estudo propõe a avaliação do impacto do design multissensorial de perfumes. Nesta pesquisa é avaliado não somente o sentido da visão mas também o olfato e tato com objetivo de mensurar o estímulo/resposta. As variáveis dependentes dos estímulos (olfato, visão e tato) consistiram no valor da marca (brand equity para o consumidor), intenção de compra, apego emocional a marca (brand attachment) e avaliação do produto multissensorial. Com amostra de 492 respondentes, um questionário estruturado foi coletado juntamente com a experienciação de perfumes e o modelo estrutural foi testado. Os resultados demostram significativos impactos do valor da marca e do produto multissensorial na intenção de compra. O valor da marca cresce com a intenção das emoções criadas pelos estímulos. O modelo explica $77 \%$ da intenção de compra. Deste modo, entende-se que este estudo contribui para o desenvolvimento do entendimento do impacto dos estímulos sensoriais nas intenções e atitudes de consumidores, bem como propõe um modelo teórico que pode ser refinado e reaplicado para avaliação sensorial em design de produtos.

Palavras-chave: Consumo de Experiência, Marketing Sensorial, Experiência de Produto 


\section{INTRODUÇÃO}

A indústria da perfumaria é referência na promoção da experiência de consumo e na construção de marcas sensoriais (LINDSTROM, 2007). No ramo da perfumaria o consumo de experiência se deu como um processo natural e hoje, segundo Achrol e Kotler (2012), a experiência de consumo tem sido apontada como uma das mudanças emergentes na fenomenologia de marketing que abrangem uma interpretação holística de seus fenômenos de domínio fundamental dos processos sensoriais humanos, enquanto bases fundamentais para explicar o consumo. O estudo do consumo de experiência promete mudar as ferramentas teóricas da análise do comportamento do consumidor acerca de conceitos cognitivos como atitudes, armazenamento de informações e teorias de recuperação para os mecanismos de representação sensorial da "realidade" e sua experiência.

Na perfumaria, estímulos sensoriais são representados através do design do frasco, da embalagem e de campanhas publicitárias para fazer esta conexão emocional da experiência com o consumidor. Em um perfume, as sensações podem ser induzidas ou exploradas através de mensagens por meio do olfato, da visão, do tato, da audição e até mesmo do paladar. Essas lembranças se tornam associações de marca quando ligadas à memória (AAKER, 1991).

O design de um produto é projetado de forma multidimensional no caso de perfumes, em sinergia sensorial com os demais atributos como olfato. Os estímulos sensoriais, além de formarem um elo emocional e de memória do consumidor com o produto, também influenciam na a percepção da qualidade, o valor e fidelização da marca (LINDSTROM 2007).

Este trabalho utiliza como objeto de pesquisa o design de perfumes (frasco e embalagem) por sua natureza de consumo de experiência ressaltando a influência dos estímulos sensoriais na intenção de compra. O objetivo desta pesquisa é avaliar empiricamente o impacto de estímulos sensoriais da visão, olfato e tato através de estímulo/resposta, de cunho subjetivo, envolvendo atributos do produto multissensorial, emoções despertadas pela marca e brand equity. Os estímulos são avaliados através de um modelo proposto utilizando escalas já validadas para identificar como os elementos sensoriais influenciam na intenção de compra da consumidora de perfumes.

\section{DESENVOLVIMENTO}

\subsection{A Dicotomia Utilitarista e Hedônica}

Holbrook e Hirschman (1982), Babin, Darden e Griffin (1994), D'Angelo (2004) e Andersson e Engelberg (2006), analisam o consumo através de uma visão dicotômica, ou seja, além de identificar o panorama racional e utilitário, eles explanam o conceito de consumo também a partir de um panorama hedônico, emocional, da compra por prazer, mesmo que muitas vezes utilizem terminologias diferentes. Chitturi et al. (2007) argumentam que há dois princípios que determinam a preferência do consumidor em relação envolvendo trocas entre os benefícios hedônicos e utilitários de design. Eles são, (1) o princípio da precedência, e (2) o princípio da dominação hedônica. O princípio da precedência motiva os consumidores a atribuir maior 
importância aos benefícios utilitários sobre os benefícios hedônicos até um mínimo limiar de funcionalidade para cumprimento das metas de prevenção. No entanto, para além deste limite mínimo de funcionalidade, o princípio da dominação hedônica motiva os clientes a atribuir maior peso à benefícios hedônicos sobre benefícios utilitários para cumprimento das metas de promoção.

\subsection{Comportamento do Consumidor e o Processo Perceptivo}

O processamento das informações pelo consumidor envolve a existência de estímulos mercadológicos, em que o objetivo é o de informar ou persuadir o consumidor à compra (BLACKWELL et al., 2001; SANTOS; BOTELHO, 2007). O processo perceptivo é um dos fatores psicológicos (juntamente com aprendizado, motivação, crenças e atitudes) pelo qual uma pessoa seleciona, organiza e interpreta as informações recebidas do ambiente (CZINKOTA, 2001). Pode-se afirmar que o processo de compra, de uma maneira geral, está intimamente ligado à percepção do consumidor, não só em relação a ele mesmo, como também, em relação ao produto ou ao serviço a ser adquirido. Assim, a percepção é algo completamente subjetivo, seletivo e limitado, isto é, pode mudar ao longo do tempo e de pessoa para pessoa (TREVISAN et al., 2003).

\subsection{Apego Emocional à Marca (Brand Attachment)}

Embora os consumidores interajam com milhares de produtos e marcas em suas vidas, eles desenvolvem uma intensa ligação emocional para apenas um pequeno subconjunto desses objetos (SCHOUTEN; MCALEXANDER, 1995). A possibilidade de que consumidores podem desenvolver fortes ligações emocionais com as marcas é associada com a teoria do apego da psicologia (BOWLBY, 1979) que sugere que o grau de ligação emocional com um objeto prevê a natureza da interação do indivíduo com o objeto. Por exemplo, indivíduos que estão fortemente ligados a uma pessoa são mais propensos a se comprometer a, investir e fazer sacrifícios para esta pessoa (BOWLBY, 1980; HAZAN; SHAVER, 1994). Analogamente, os consumidores ligados emocionalmente a uma marca podem prever seu compromisso com ela e sua disposição de fazer sacrifícios financeiros, a fim de obtê-lo, como fidelização e disposição para pagar preço premium.

Thomson, Maclnnis e Park (2005) afirmam que o apego emocional a marca (brand attachment) pode ser definido como um construto que descreve a força da ligação que conecta o consumidor com a marca, sendo o apego fundamental porque pode afetar comportamentos que promovem a rentabilidade da marca e o lifetime value dos consumidores.

\subsection{Experiência de Consumo}

Segundo Achrol e Kotler (2012), o estudo do consumo de experiência promete mudar as ferramentas teóricas da análise do comportamento do consumidor acerca de conceitos cognitivos como atitudes, armazenamento de informações e teorias de recuperação para os mecanismos de representação sensorial da "realidade" e sua experiência. O processo fundamental no marketing é o consumo, e os conceitos elementares de consumo são a satisfação, valor e utilidade. Holbrook (2006) aponta que a ideia de o consumo de experiência é, no fundo, valor para o consumidor. E antes disso, o economista Lawrence Abbott já afirmava que as pessoas realmente desejam 
não são produtos, mas satisfazer experiências “(...) as pessoas querem produtos porque eles querem a experiência trazendo serviços que esperam que os produtos vão render" (ABBOTT, 1955, p. 40).

Neste sentido, as experiências podem ser consideradas acontecimentos individuais que ocorrem como resposta a algum estímulo. São o resultado de uma observação direta e /ou de participação nos acontecimentos reais, imaginários ou virtuais (SCHMITT, 2002).

\subsection{Experiência de Produto}

Experiências de Produto ocorrem quando o consumidor interage com os produtos, por exemplo, quando os consumidores procuram por produtos e examinam e os avaliam $(\mathrm{HOCH}, 2002)$. A experiência com o produto pode ser direta, quando existe um contato físico com o produto ou indireta, quando um produto é apresentado ou virtualmente em publicidade (HOCH; HA, 1986; KEMPF; SMITH, 1998).

Experiências também podem ocorrer quando os consumidores consumem e usam produtos. Em resumo, surgem experiências em uma variedade de configurações. A maioria das experiências ocorrem diretamente quando os consumidores vão as compras, compram e consomem produtos (HOLBROOK; HIRSCHMAN, 1982).

O processamento do julgamento destas experiências começam com uma combinação dos sentidos da visão, tato, olfato e auditiva e/ou dados de gosto que são canalizados para o registro sensorial (LANDWEHR; WENTZEL; HERRMANN, 2013). Os sentidos captam informações do ambiente externo, estas informações são levadas ao sistema límbico cerebral e reprocessados através de neuro-transmissores que permitem respostas como gosto / não gosto. Neste âmbito, esta pesquisa se aproxima da neurociências.

\subsection{Marketing Sensorial}

O objetivo do marketing sensorial é captar a atenção dos cinco sentidos, causando prazer estético, emoções ou excitação nos consumidores. Para destacar os produtos por meio do apelo sensorial, é preciso considerar quais os estímulos mais apropriados para criá-lo. Para motivar os clientes, é preciso identificar os princípios do processo. Para prover valor, é preciso entender as conseqüências do apelo sensorial. Adequadamente administrado, o marketing dos sentidos cria experiências sensoriais poderosas que diferenciam companhias e produtos, motivam consumidores e agregam valor (SCHMITT, 2002).

Conforme relatado por Lindstrom (2007) quanto mais pontos sensoriais forem estimulados ao construir marcas, maior será o número de memórias sensoriais ativadas e quanto maior o número de memórias sensoriais ativadas, mais forte será a adesão do consumidor à marca.

\section{METODOLOGIA}

Em pesquisas para avaliar a experiência, os entrevistados são normalmente solicitados a refletir sobre uma combinação de experiências de produtos diretos e indiretos para investigar como a combinação de produtos afeta julgamentos, atitudes, preferências, intenção de compra, e recordação (HOCH e DEIGHTON 1989; HOCH e HA 1986; HUFFMAN e HOUSTON, 1993). 
Para avaliar os sentidos do olfato, tato e visão, os respondentes experienciaram cada sentido isoladamente de perfumes e em seguida responderam ao questionário. As embalagens eram tocadas dentro de recipiente sem visão. O odor era sentido em recipientes sem marca, em forma de conta gotas. Já a visão era realizada em uma caixa que se assemelha a um oratório. Os respondentes recebiam como incentivo a participação um ticket para lanche. A coleta foi realizada em um período de 5 dias úteis em horários distintos (manhã, tarde e noite). O experimento foi realizado em ambiente de laboratório e foi organizado em 16 grupos com cerca de 30 componentes do sexo feminino usuárias de perfumes. No total obteve-se 492 questionários respondidos. O questionário foi aplicado utilizando escalas do tipo Likert de 10 pontos com âncoras 0 (discordo totalmente) a 10 (concordo totalmente) (SPECTOR, 1992).

\section{Caracterização da amostra}

No que se refere a renda familiar dos 492 respondentes, observa-se uma boa distribuição nos diversos extratos de renda, com uma maior frequência nos extremos.

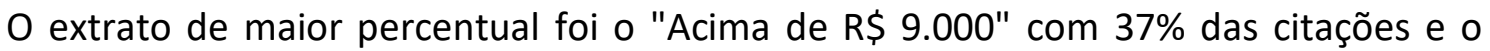
extrato "Até $\mathrm{R} \$ 3.000$ " com $19 \%$. Os outros extratos apresentaram de $4 \%$ a $9 \%$ de citações.

A amostra foi composta de estudantes de uma universidade de Minas Gerais, sendo $94 \%$ solteiros, e que em média os entrevistados possuem 22 anos com desvio padrão foi de 4 anos.

\section{Operacionalização da Mensuração dos Constructos}

Para avaliação dos estímulos sensoriais do olfato, visão e tato foi utilizada a escala PANAS de Allen e Janiszewski (1989). A escala Overall Brand Equity proposta por Yoo, Donthu e Lee (2000) foi utilizada para medir o valor da marca para o consumidor (brand equity). Já o apego emocional à marca (brand attachment) foi mensurado pela escala de Thomson, MacInnis e Park (2005). Brakus, Schmitt e Zarantonello, (2009) desenvolveram uma escala de produto multissensorial em que são avaliadas quatro dimensões: sensorial, afetiva, intelectual e comportamental. Em seus estudos, os autores mostraram que a escala é confiável e válida.

Como se tratavam de três variáveis independentes dicotômicas e quatro variáveis dependentes escalares, optou-se por realizar uma MANOVA por ser a mais adequada ao caso. Ela pode ser considerada como uma análise de três fatores, sendo mais indicada nesse desenho de pesquisa, uma vez que, a realização de diversos Testes $t$ aumentariam a chance de cometer o erro tipo I (HAIR et al., 2009).

\section{Análise Preliminar}

A análise preliminar constou de analises de valores extremos, outliers uni e multivariados e linearidade, de modo a proceder as etapas seguintes da analise.

\section{Validade e Confiabilidade das mensurações}

No caso dos construtos que foram tratados na literatura como unidimensionais optou-se por realizar uma AFE para cada um deles, utilizando a análise de componentes principais como método de extração e como método de rotação foi o varimax. 
Método de Equações Estruturais - Análise fatorial confirmatória dos construtos e teste do modelo proposto

Após definida a dimensionalidade dos construtos foi dado prosseguimento à análise, de modo a realizar a AFC dos construtos e testar o modelo proposto. Para tanto foi utilizado o Método de Equações Estruturais (MEE). Ele foi imprescindível no teste de algumas das hipóteses desta pesquisa por permitir trabalhar com construtos, testar relações de dependência simultâneas, e levar em conta o erro de mensuração (HAENLEIN, KAPLAN, 2004; MACKENZIE, 2001).

Para tanto, podem ser utilizados dois grandes métodos de estimação dos parâmetros no MEE: o primeiro pode ser denominado de CB-SEM e é baseado na matriz de covariância; já o segundo é denominado de PLS-SEM e é baseado na variância (HAENLEIN, KAPLAN, 2004).

O método Generalized Least Square (GLS), que pertence ao CB-SEM lida bem com a falta de normalidade dos dados, caso a amostra seja suficientemente grande para o modelo testado.

\section{Outer Model - Validade convergente, discriminante e confiabilidade}

Neste tópico são apresentados os resultados do Outer Model dos construtos, que compreende a validade convergente, a validade discriminante e a confiabilidade dos mesmos. Como a pesquisa possuía um construto de segunda ordem, o Produto multisensorial, será mostrado primeira os resultados dos construtos de primeira ordem para então mostrar os resultados do construto de segunda ordem citado. De modo a testar a validade convergente dos construtos, foram utilizados dois critérios: 0 proposto por Bagozzi, Yi e Philips (1991) e o proposto por Fornell e Lacker (1981), o que foi realizado com sucesso para todos os constructos e itens. Para verificar a validade discriminante foi adotado o critério de Fornell e Lacker (1981) que consiste em comparar a correlação elevada ao quadrado de todos os pares de construtos do modelo com as AVEs dos respectivos pares. Nesse sentido, caso a correlação ao quadrado for inferior à AVE dos dois construtos comparados pode-se atestar a validade discriminante dos mesmos. Foi possível atestar a validade discriminante uma vez que para todos os pares de construtos a correlação ao quadrado entre eles é menor que a AVE de ambos os construtos. Os Alphas de Cronbach apresentaram valores acima de 0.6, com AVE e CC acima de 0,50, o que atesta a confiabilidade da mensuração.

\section{Inner Path Model - Validade nomológica e ajuste do modelo}

Neste tópico da pesquisa será testado o modelo proposto. Para tanto, é importante verificar a validade nomológica e o ajuste do modelo, por meio do Inner Path Model.

A FIG. 1 exibe o teste do Modelo Proposto pela pesquisa. O construto Produto multisensorial foi tratado como de segunda ordem reflexivo, composto de quatro subdimensões: afetiva, comportamental, intelectual e sensorial. No caso do Produto multisensorial $59 \%$ das suas variações foram explicadas pelo Olfato, Tato e Visão. Todos apresentaram impacto positivo e estatisticamente significativo ao nível de $1 \%$ ou 5\%. O Olfato apresentou a carga de maior magnitude, da ordem de 0,66. Já o Tato apresentou a carga de menor magnitude, da ordem de 0,08 . 
O construto Emoções despertadas pela marca apresentou um $\mathrm{R}^{2}$ de $39 \%$, que são explicadas também pelo Olfato, Tato e Visão. O Olfato apresentou um impacto de 0,46 (sig. $<1 \%$ ), o Tato de 0,08 (sig. $<5 \%$ ) e a Visão de 0,30 (sig. $<1 \%$ ). Já o construto Brand Equity apresentou um $\mathrm{R}^{2}$ de $49 \%$, que são explicadas pelo Olfato, Tato, Visão, Produto multissensorial e Emoções despertadas pela marca. De todos apenas o Produto multissensorial apresentou um impacto positivo e estatisticamente significativo ao nível de $1 \%$ da ordem de 0,61 . Por fim tem-se a Intenção de compra que $77 \%$ das suas variações foram explicadas pelo Olfato, Tato, Visão, Produto multissensorial e Brand Equity. Todos os construtos exógenos nesse caso apresentaram impacto estatisticamente significativo, sendo que o de maior magnitude foi do Produto multissensorial ( $\beta$ de 0,47 , sig. $<1 \%$ ) e o de menor magnitude foi o Tato ( $\beta$ de $-0,05$, sig. $<5 \%$ ).

Figura 1 - Teste do Modelo Proposto na pesquisa.

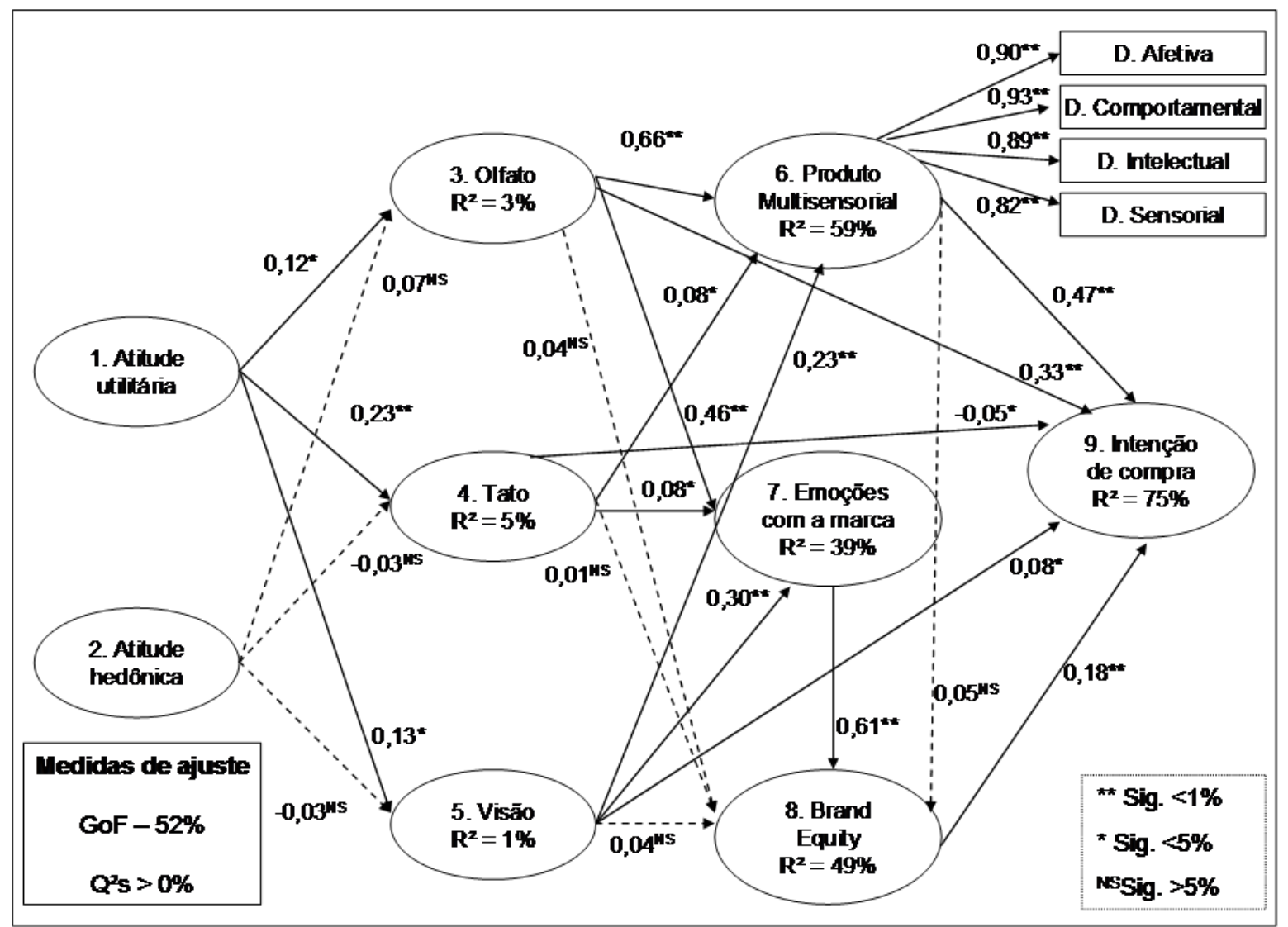

Fonte: Elaborado pelo autor

Legenda: setas pontilhadas indicam que o construto exógeno não apresentou impacto estatisticamente significativo ao nível de $5 \%$ no construto endógeno.

No que tange ao ajuste do modelo, todos os construtos endógenos apresentaram um $\mathrm{Q}^{2}$ superior a $0 \%$ indicando uma adequada mensuração dos mesmos. Já o GoF foi de $52 \%$. Ainda que não exista um critério de avaliação esse valor pode ser considerado elevado, o que significa que o modelo possui um bom ajuste. 
A TAB. 1 apresenta o resultado dos pesos do Modelo Proposto em forma de tabela, conforme foi exibido na FIG. 1.

Tabela 1 - Resultado dos pesos do Modelo Proposto.

\begin{tabular}{|c|c|c|c|c|c|c|c|}
\hline Construto exógeno & Construto endógeno & Amostra & Pop. & Desv. & Erro & Valor T & Sig. \\
\hline 1. Atitude utilitária & Olfato & 0,12 & 0,12 & 0,06 & 0,06 & 1,97 & $2,5 \%$ \\
\hline 2. Atitude hedônica & $\mathrm{R}^{2}=3 \%$ & 0,07 & 0,06 & 0,05 & 0,05 & 1,28 & $10,0 \%$ \\
\hline 1. Atitude utilitária & Tato & 0,23 & 0,24 & 0,06 & 0,06 & 3,96 & $0,0 \%$ \\
\hline 2. Atitude hedônica & $R^{2}=5 \%$ & $-0,03$ & $-0,03$ & 0,06 & 0,06 & 0,42 & $33,8 \%$ \\
\hline 1. Atitude utilitária & Visão & 0,13 & 0,14 & 0,05 & 0,05 & 2,52 & $0,6 \%$ \\
\hline 2. Atitude hedônica & $R^{2}=1 \%$ & $-0,03$ & $-0,03$ & 0,06 & 0,06 & 0,51 & $30,6 \%$ \\
\hline 3. Olfato & & 0,66 & 0,66 & 0,02 & 0,02 & 27,93 & $0,0 \%$ \\
\hline 4. Tato & $\begin{array}{l}\text { 6. Produto Multisensorial } \\
R^{2}=59 \%\end{array}$ & 0,08 & 0,08 & 0,03 & 0,03 & 2,35 & $1,0 \%$ \\
\hline 5. Visão & & 0,23 & 0,23 & 0,04 & 0,04 & 6,37 & $0,0 \%$ \\
\hline 3. Olfato & & 0,46 & 0,46 & 0,04 & 0,04 & 12,50 & $0,0 \%$ \\
\hline 4. Tato & $\begin{array}{l}7 . \\
R^{2}=39 \%\end{array}$ & 0,08 & 0,08 & 0,04 & 0,04 & 1,99 & $2,4 \%$ \\
\hline 5. Visão & & 0,30 & 0,30 & 0,04 & 0,04 & 7,37 & $0,0 \%$ \\
\hline 3. Olfato & & 0,04 & 0,05 & 0,05 & 0,05 & 0,83 & $20,3 \%$ \\
\hline 4. Tato & & 0,01 & 0,01 & 0,04 & 0,04 & 0,32 & $37,3 \%$ \\
\hline 5. Visão & $\begin{array}{l}\text { 8. } \\
R^{2}=49 \%\end{array}$ & 0,04 & 0,04 & 0,04 & 0,04 & 1,04 & $15,0 \%$ \\
\hline 6. Produto Multisensorial & & 0,05 & 0,06 & 0,06 & 0,06 & 0,95 & $17,1 \%$ \\
\hline 7. Emoções & & 0,61 & 0,61 & 0,05 & 0,05 & 12,93 & $0,0 \%$ \\
\hline 3. Olfato & & 0,33 & 0,33 & 0,04 & 0,04 & 9,28 & $0,0 \%$ \\
\hline 4. Tato & & $-0,05$ & $-0,06$ & 0,03 & 0,03 & 1,87 & $3,1 \%$ \\
\hline 5. Visão & $\begin{array}{l}\text { 9. Intenção de compra } \\
R^{2}=75 \%\end{array}$ & 0,08 & 0,08 & 0,03 & 0,03 & 2,82 & $0,2 \%$ \\
\hline 6. Produto Multisensorial & & 0,47 & 0,47 & 0,04 & 0,04 & 12,55 & $0,0 \%$ \\
\hline 8. Brand Equity & & 0,18 & 0,18 & 0,03 & 0,03 & 5,97 & $0,0 \%$ \\
\hline
\end{tabular}

Fonte: Dados da pesquisa

Observações: a) Amostra: é o peso padronizado obtido para amostra completa; b) Pop.: é o peso médio obtido na população; c) Desv.: é o desvio padrão da estimativa; d) Erro: é o erro estimado da estimativa; e) Valor T: é a razão entre o peso não padronizado pelo seu erro padrão.

\section{CONCLUSÕES FINAIS}

Pesquisas sobre experiências do consumidor são recentes e escassas, em especial aquelas que são resultado de estímulos reais, pela dificuldade e custos de execução. Por outro lado, cabe ressaltar recente interesse em compreender as relações entre estímulos sensoriais, avaliação de marcas e produtos, o que tem sido fruto de estudos mais recentes de neuromarketing. Nesta pesquisa 492 respondentes 
foram expostos a estímulos individuais em um laboratório, onde viam produtos reais sem marcas, cheiravam (sem ver os produtos ou toca-los) e tocavam estes produtos em caixas escuras sem vê-los ou cheirá-los, antes de responder o questionário (experimento fatorial seguido de levantamento).

No modelo hipotético, buscou-se identificar os impactos da intensidade dos estímulos sensoriais nas avaliações multissensoriais do produto, em emoções com a marca bem como com a avaliação da marca (brand equity para o consumidor) e intenções de compra. Hipoteticamente os estímulos sensoriais tiveram hipóteses testadas sobre seus impactos nos constructos mediadores (produto sensorial, emoções com a marca e brand equity), bem como na intenção de compra, buscado assim entender como esta cadeia nomológica se comporta na prática na mente do consumidor.

Observou-se, conforme constatações empíricas, uma intermediação entre os estímulos sensoriais e o valor da marca, realizada pelas emoções com a marca. Em outras palavras, os resultados sugerem que os estímulos sensoriais provocam emoções, e estas por sua vez impactam o valor da marca, demonstrando o papel das emoções na geração de brand equity (não foram significativos os impactos dos estímulos sensoriais diretamente no valor da marca, mas somente quando mediados pelas emoções coma marca). No caso de perfumes, verificou-se que olfato e visão causam mais emoções positivas e por conseguinte, contribuem indiretamente para uma melhor avaliação das marcas.

Os resultados sugerem ainda que a avaliação do produto (escala produto multissensorial de Brakus, Schmitt e Zarantonello (2009) é afetada pelos estímulos de forma integrada, em especial olfato e visão. O modelo explicou $75 \%$ da intenção de compra, o que de certa forma demonstra propriedades importantes para entendimento das relações entre estímulos sensoriais, marcas, produtos, emoções e intenções de compra.

Cabe ressaltar que neste estudo os consumidores viram, cheiraram e tocaram os produtos, e possivelmente por este motivo, o construto produto multissensorial foi determinante na decisão seguido do brand equity. Porém observou-se que o produto em si não impactou o valor da marca conforme hipotetizado, mas as emoções criadas pela experiência, o que sugere que aparentemente o consumidor cria em sua mente um conceito independente de produto e de marca, e de certa forma consegue separar estes conceitos, ou pelo menos os impactos que recebem de suas experiências sensoriais.

Acredita-se que o modelo proposto e resultados inovam e contribuem para o entendimento das relações entre elementos sensoriais, marcas, emoções, produtos e intenção de compra dos consumidores, gerando subsídios para estudos e experimentos posteriores trazem conhecimento sobre o fenômeno de escolha dos consumidores, em especial para produtos que envolvem impactos sensoriais como é o caso de perfumes.

\section{REFERÊNCIAS}


ABBOTT, L. Quality and competition. New York: Columbia Univ. Press, 1955.

ABHIPEC, Anuário de 2012 da Associação Brasileira da Indústria de Higiene Pessoal, Perfumaria e Cosméticos, 3a edição: 2012. Disponível em

$<$ http://www.abihpec.org.br/wpcontent/uploads/2012/12/ABIHPEC_2012_internet.pd f>. Acesso em: 20 fev 2013.

ACHROL, R. S.; KOTLER, P. Frontiers of the marketing paradigm in the third millennium in: Journal Academy of Marketing Science, p. 35-52, 2012

ALLEN, C.T.; JANISZEWSKI, C. A. Assessing the Role of Contingency Awareness in Attitudinal Conditioning with Implications for Advertising Research in: Journal of Marketing Research, v. 26, p. 30-43, 1989.

ANDERSSON, P.; ENGELBERG, E. Affective and rational consumer choice modes: The role of intuition, analytical decision-making, and attitudes to money. Working Paper in Business Administration. n.13, 2006. Disponível em:<

http://swoba.hhs.se/hastba/papers/hastba2006_013.pdf>. Acesso em: 03 jun. 2011.

BABIN, B. J., DARDEN, W. R.; GRIFFIN, M. Work and/or fun: Measuring hedonic and utilitarian shopping value in: Journal of Consumer Research, v. 20, p. 644-656, 1994.

BAGOZZI, R. P.; YI, Y.; PHILLIPS, L. W. Assessing construct validity in organizational research in: Administrative Science Quarterly, v. 36, p. 421-458, 1991.

BARWISE, P. Brand equity: Snark or Boojum? in: International Journal of Marketing Research v. 10, n.1, p. 93-104, 1993.

BATRA, R.; AHTOLA, O. T. Measuring the hedonic and utilitarian sources of consumer attitudes in: Marketing Letters, v. 2, p. 159-170, 1990.

BLACKWELL, R. D.; MINIARD, P. W.; ENGEL, J. F. Consumer behavior. 9. ed. Orlando: Harcourt, 2001.

BOWLBY, J. Loss: Sadness and depression. New York: Basic Books, 1980.

BOWLBY, J. The making and breaking of affectional bonds. London: Tavistock, 1979.

BRAKUS, J. J.; SCHMITT, B. H.; ZARANTONELLO, L. Brand Experience: What Is It? How Is It Measured? Does It Affect Loyalty? in: Journal of Marketing, v. 73, p. 52-68, 2009.

CHITTURI, R.; RAGHUNATHAN, R.; MAHAJAN V. Form Versus Function:How the Intensities of Specific Emotions Evoked in Functional Versus Hedonic Trade-Offs Mediate Product Preferences in: Journal of Marketing Research: v. 44, No. 4, p. 702714, 2007.

CZINKOTA, M. R. Marketing: As Melhores Práticas. Porto Alegre: Bookman, 2001.

D’ANGELO, A. C. Valores e significados do consumo de produtos de luxo. 2004.

Dissertação (Mestrado Acadêmico em Administração) - Universidade Federal do Rio

Grande do Sul. Programa de Pós-Graduação em Administração. Escola de

Administração, Porto Alegre, 2004. Disponível em:<

http://hdl.handle.net/10183/4845>. Acesso em: 02 jun. 2011.

DIAMANTOPOULOS, A.; SIGUAW, J. A. Formative versus Reflective Indicators in Organizational Measure Development: A Comparison and Empirical Illustration in:

British Journal of Management v. 17, n.4, p. 263-282, 2006. 
FORNELL, C.; LARCKER, D. F. Evaluating Structural Equation Models with Unobservable Variables and Measurement Error in: Journal of Marketing Research, v. 18, p. 39-50, 1981.

HAENLEIN, M.; KAPLAN, A.M. A beginner's guide to partial least squares (PLS) analysis in:Understanding statistics, v. 3, n. 4, p. 283-297, 2004.

HAIR, J. F.; BLACK, W. C,; BABIN, B. J.; ANDERSON, R. E.; TATHAM, R. L. Análise Multivariada de Dados. Porto Alegre: Bookmam, 2009.

HAZAN, C.; SHAVER, P. R. Attachment as an organizational framework for research on close relationships in: Psychological Inquiry, v. 5, 1994.

$\mathrm{HOCH}$, S. J. Product Experience Is Seductive in: Journal of Consumer Research, v. 29 p. 448-54, 2002

$\mathrm{HOCH}$, S. J.; HA, Y. Consumer Learning: Advertising and the Ambiguity of Product Experience in: Journal of Consumer Research, v. 13, p. 221-33, 1986.

HOLBROOK, M. B. Rosepekiceciveci vs. CCV: The Resource-Operant, Skills-Exchanging, Performance-Experiencing, Knowledge-Informed, Competence-Enacting, Co-producerInvolved, Value-Emerging, Customer-Interactive View of Marketing versus the Concept of Customer Value: "I Can get it for you Whosale" in: LUSCH, R. F.; VARGO, S. L. (Eds.) The service-dominant logic of marketing: Dialog, debate and directions. Armonk: M. E. Sharpe. p. 208-223, 2006.

HOLBROOK, M. B.; HIRSCHMAN, E. C. The Experiential Aspects of Consumption: Consumer Fantasies, Feelings and Fun in: Journal of Consumer Research, v. 9, p. 132140, 1982.

KEMPF, D. S.; SMITH R. E. Consumer Processing of Product Trial and the Influence of Prior Advertising: A Structural Modeling Approach in: Journal of Marketing Research, $v$. 35, p. 325-38, 1998.

LANDWEHR J. R.; WENTZEL D.; HERRMANN, A. Product Design for the Long Run: Consumer Responses to Typical and Atypical Designs at Different Stages of Exposure in: Journal of Marketing, v. 77, no. 5, p. 92-107, 2013

LINDSTROM, M. Brand Sense: a marca multissensorial. Porto Alegre: Bookman, 2007. MACKENZIE, S. B. Opportunities for Improving Consumer research through latent Variable Structural Equation Modeling. Journal of Marketing Research, 28, n. 1, p.159166, 2001.

MALHOTRA, N. K. Pesquisa de marketing: uma orientação aplicada. 4. ed. Porto Alegre: Bookman, 2006.

OLIVEIRA, C. A.; Inovação do Produto e do Processo, Belo Horizonte: Ed. de Desenvolvimento Gerencial, 2000.

SANTOS, J. H. F.; BOTELHO, D. Analise Comparativa de Preços: estudo de variáveis influentes na percepção de vantagem de compra in: Encontro Nacional da Associação Nacional de Pós-Graduação e Pesquisa em Administração, ENANPAD, 31, Rio de Janeiro, Anais... Rio de Janeiro: ANPAD, 2007.

SCHMITT, B. Marketing Experimental. São Paulo: Nobel, 2002. 
SCHOUTEN, J. W.; MCALEXANDER, J. H. Subcultures of consumption: An ethnography of the new bikers. Journal of Consumer Research, v. 22, p. 43-61, 1995.

SPECTOR, P. E. A Consideration of the validity and meaning of self-report measures of job conditions in: COOPER, C. L.; ROBERTSON, I. T. (Eds) International Review of Industrial and Organizational Psychology, West Sussex: John Wiley, 1992.

THOMSON, M.; MACINNIS, D. J.; PARK, C. W. The Ties That Bind: Measuring the Strength of Consumers' Emotional Attachments to Brands in: Journal Of Consumer Psychology, v. 15, n. 1, p. 77-91, 2005.

TREVISAN, R.; SAMPAIO C. H.; PERIN, M. G. Estratégia de lançamento de Novos Produtos na Indústria Automobilística: o Caso do Volkswagen Novo Pólo in: Congresso Latinoamericano de Estratégia - Estratégias para la Era de la Complijidad y la Imaginación, 16, Lima, Anais... Lima: Congresso Latinoamericano de Estratégia, 2003.

VERAMENDI, M., HERENCIA, P., ARES, G. Perfume Odor Categorization: To What Extent Trained Assessors and Consumers Agree? in: Journal of Sensory Studies, v. 28, n. 1, p. 76-89, 2013.

YOO, B.; DONTHU, N.; LEE, S. An examination of selected marketing mix elements and brand equity, Journal of the Academy of Marketing Science, v. 28, p. 195-211, 2000. 